\title{
Rheological considerations on polymer-based engine lubricants: viscosity index improvers versus thickeners - generalized Newtonian models
}

http://dx.doi.org/10.1080/10402004.2017.1346154

\author{
Pauline Cusseau $^{1,2}$, Nathalie Bouscharain ${ }^{1}$, Laetitia Martinie ${ }^{1}$, David Philippon ${ }^{1}$, Philippe \\ Vergne $^{1}$, Fanny Briand ${ }^{2}$
}

1 Univ Lyon, INSA Lyon, CNRS, LaMCoS - UMR5259, F-69621 Villeurbanne, France

2 TOTAL, Centre de Recherche de Solaize, BP 22, F-69360 Solaize, France

\begin{abstract}
The development of high-performance lubricants to decrease engine friction and then reduce fuel consumption remains a major challenge for oil manufacturers. Viscosity Index Improvers (VIIs) are additives used for decades to reduce the dependency of the lubricant's viscosity on temperature, to maintain an acceptable lubrication in harsh conditions. Distinction between VII and thickener in realistic engine conditions is of primary interest for oil manufacturers in order to optimize the formulation process. In this context, the rheological study can provide a clear insight on the actual effect of such polymeric additives. The behavior of simplified automotive lubricant was investigated at different temperature, high pressure and high shear stress and a modelling of their properties is proposed. Various polymers of different molecular weights and conformations were mixed in a hydrocracked mineral base oil. The viscosity variations with temperature, pressure and shear stress, obtained from specific rheometers, were then represented by different models, namely a Vogel-Fulcher-Tamman expression, a modified William-Landel-Ferry correlation and a Carreau-Yasuda equation. Different rheological responses were observed and allowed the distinction between VIIs and thickeners, upon different temperature and pressure conditions. The hydrodynamic radii analysis provided an explanation of the rheological responses but also highlighted the limits of the Carreau-Yasuda equation when applied to VIIs. Therefore the Zhang expression, based on the Maxwell model and applied here to low viscoelastic solutions, is proposed as an appropriate alternative.
\end{abstract}




\section{KEYWORDS}

Polymeric additives, Viscosity Index Improvers, Viscosity Thickeners, Rheology, Viscosity-Pressure dependence, Viscosity-Temperature dependence, Generalized Newtonian Model

\section{INTRODUCTION}

Reducing fuel consumption in transportation is a main challenge of the automotive industry, given the current context of global warming. Increasing energy efficiency by mainly limiting car engines losses have become essential. About $10 \%$ of the fuel energy is indeed dissipated by engine friction, which must be reduced to decrease fuel consumption (1). The development of high-performance lubricants is a mean to achieve friction reduction in car engines, by maintaining a thin film of lubricant between the rubbing surfaces in relative motion.

Lubrication properties are determined relatively by the fluid rheological behavior. The latter may be adjusted by the addition of viscosity index improvers (VIIs). The VIIs are high molecular weight polymers used to reduce the dependency of the lubricant's viscosity on temperature, in order to achieve an acceptable lubrication at high temperature, without experiencing excessive frictional losses at low one. These polymers have indeed little effect at low temperature due to their globule-like conformation. This globular state is acknowledged as the standard description, even though it may be arguable (2). As the temperature increases, the polymer coils change from rather small globules to a more open configuration and thus exhibit a greater thickening effect $(3,4)$. In other words, the variation of the polymer coils dimensions plays an important role in the VIIs efficiency. Actual viscosity index improvers can be then distinguished from thickeners, whose coils are not significantly affected by temperature changes and whose addition results in a uniform viscosity increase in a viscosity-temperature chart. The VIIs efficiency is measured by the viscosity index (VI), a standard value quantifying the viscosity variations upon temperature (5), or alternatively by the thickening factor $Q$ (6).

Three major families of VIIs are usually considered (7): olefin copolymers (OCP), hydrogenated dienestyrene copolymers - including for instance poly-isoprene styrene hydrogenated (PISH) - and polyesters like poly-alkylmethacrylates (PAMA). The main molecular chain of these polymers is generally rather flexible: these materials are therefore shear sensitive and responsible of the shear-thinning behavior of the lubricants $(8,9)$. Different rheological responses regarding the temperature, pressure and shear stress influences are then expected, according to the VII's structure and conformation and their variations with 
the above-mentioned parameters. As stated previously, VIIs efficiency is enhanced by the polymer coil size expansion with increasing temperature. It appears from the literature that long polyester chains (like in PAMA) can exhibit excellent VII additive performance when added to a base oil: Covitch and Trickett (10) highlighted the swelling of PAMA coils with temperature at atmospheric pressure using SmallAngle Neutron Scattering (SANS), whereas in their work OCP coils showed no variation and behaved more like thickeners, also called viscosity modifiers. However, the impact of pressure and shear stress on the coil expansion was not investigated in (10).

High pressure, high shear stress, high temperature encountered in engine parts have a strong impact on the properties of lubricants. These severe conditions may lead to the elastohydrodynamic lubrication (EHL) regime which is highly desired to insure the separation of the contacting surfaces while assuming a rather low friction. Progresses in quantitative EHL are the results of accurate measurements and predictions of the rheological and physical lubricant's properties $(11,12)$ as well as of the successful comparisons $(12,13)$ between predicted and measured tribological quantities like film thickness and friction. Therefore, establishing valid rheological models for polymer-base oil solutions is of main importance to determine their viscosity close to engine. These models can also be included in elastohydrodynamic (EHD) simulations to predict film thickness without using a full-scale bench test (14). This approach is time and cost saving for oil manufacturers and the present work follows the same approach.

The goal here is i) to quantify the effect of temperature, pressure and shear stress on the lubricants' response when submitted to realistic engine conditions and ii) to highlight the actual performance of the different polymers studied in simplified lubricants, composed of a VII and the base oil only. The materials and devices are first described, followed by high pressure rheological measurements. The impact of temperature, pressure and shear stress on the VIIs properties is then explained by the molecular structure of the polymers. These results also help to highlight some limitations of the Carreau-Yasuda model in the case of VII. An alternative, the Zhang model, is then proposed. Finally, the comparison between these two models reveals the possibility to apply the Zhang expression to weakly viscoelastic solutions.

\section{MATERIALS AND EXPERIMENTAL}

\section{Materials}

In this work, mixtures of base oil and Viscosity Index Improvers (VIIs) were considered as simplified automotive lubricants. All materials were provided by TOTAL and were property of TOTAL. The base 
oil studied was a hydrocracked mineral base oil from group III (Yubase 4+), according to API (American Petroleum Institute) classification. Several polymers with different chemistry - PAMA (polyalkylmethacrylate), PISH (polyisoprene styrene hydrogenated), OCP (olefin copolymer) - and conformations - comb, star, linear (noted -C, -S and -LE)) - were studied and details can be found in Table 1. The given weight average molecular weights $M_{w}$ and polydispersity Index (PDI) were obtained through triple detection size exclusion chromatography (SEC).

Commercial products were used, meaning that all polymers were previously mixed with an extender oil, whose chemical properties were close to that of the mineral base oil used here. The selection of raw material allows the comparison between the different structures and provides solutions closer to commercial formulations.

In this study, all solutions were formulated at a HTHS (High Temperature High Shear) iso-viscosity value of $2.6 \mathrm{mPa}$.s. The HTHS test leads to the measure of the dynamic viscosity at $150{ }^{\circ} \mathrm{C}$ and at elevated shear rate $\left(10^{6} s^{-1}\right)$, according to the ASTM standard D4741 (15). These specifications are representative of the engine conditions encountered by the lubricant and help in determining whether an estimate of the film thickness gives an acceptable value, considering for instance the roughness of the surfaces. Therefore, all solutions have different polymer concentrations, given in Table 1. Both PISH-S and OCP-LE solutions are formulated at comparable concentration, whereas the PAMA-C is introduced in bigger quantity. However, all solutions are in the diluted regime, at standard ambient temperature and pressure conditions.

\section{Atmospheric pressure rheometer}

A Physica MCR 301 rheometer (Anton-Paar) with a coaxial cylinders geometry was used to measure the dependency of the polymer-base oil solution's viscosity on temperature. This latter was varied from -20 to $150{ }^{\circ} \mathrm{C}$ and the shear rate from $0.3 \mathrm{~s}^{-1}$ to $3000 \mathrm{~s}^{-1}$ (equivalent to shear stress of about $10^{-2}$ to $10^{2} \mathrm{~Pa}$ ). The uncertainty on the viscosity is always below $2 \%$.

\section{High pressure rheometers}

Two devices were used to study the viscosity under high pressure: a falling body viscometer and a Couette rheometer.

The first apparatus, fully described in (16) measured the viscosity of the simplified lubricants over a wide pressure range up to $800 \mathrm{MPa}$. Temperature was varied from 40 to $120^{\circ} \mathrm{C}$ and shear stress from 1.5 to $70 \mathrm{~Pa}$. The statistical uncertainty on the viscosity was always below $5 \%$. 
The second instrument was a Couette rheometer, depicted in (17), and allowed to explore the shear stress influence on viscosity at pressures from $200 \mathrm{MPa}$ to $400 \mathrm{MPa}$. Temperatures ranged from $30{ }^{\circ} \mathrm{C}$ to 60 ${ }^{\circ} \mathrm{C}$. Three cylinders sets were used here to cover a large range of shear stress from $0.5 \mathrm{kPa}$ up to $100 \mathrm{kPa}$. The statistical uncertainty on the viscosity values was below $10 \%$.

\section{INFLUENCE OF TEMPERATURE AND PRESSURE ON THE VIIs' RHEOLOGY VII behavior at atmospheric pressure}

Study of the temperature's effect on the viscosity at atmospheric pressure and low shear stress is the first step to differentiate VIIs from thickeners. These latter are commonly called VII, however their properties do not comply with the desired effect. The temperature dependence of the low shear viscosity $\eta_{0}$ was acquired using the Physica MCR 301 rheometer. Many models based on the Arrhenius equation have been proposed to describe this viscosity response at atmospheric pressure $(18,19)$. However, the VogelTamman-Fulcher (VTF) model (20) is more suitable to base oil applications as shown in (10) and was thus chosen here to fit the experimental data:

$$
\eta_{0}(T)=\eta_{\infty} \exp \left(\frac{D_{F} T_{\infty}}{T-T_{\infty}}\right)
$$

where $\eta_{\infty}$ is the viscosity extrapolated to infinite temperature, $D_{F}$ the fragility parameter, $T$ the temperature and $T_{\infty}$ the Vogel temperature at which the viscosity diverges.

Results for the base oil, PAMA-C, PISH-S and OCP-LE solutions are presented in Fig. 1. Each polymer added thickens the solution. However, their thickening contribution is not the same regarding the temperature. The desired effect of a true VII is to present variation upon temperature of its thickening power, which must increase at high temperature. This response is reflected as a slope change at high temperature, with a reduced slope compared to the base oil one. Other variations of the thickening power define here a thickener. Fig. 1 (left and right) highlight the different properties of each polymer family. The PAMA-C solution presents the strongest VII effect, with a viscosity close to the base oil's one at low temperature and an important viscosity improvement at high temperature. This effect is much less noticed for the PISH-S solution, but this latter can still be considered as a viscosity index improver. However, the OCP-LE solution exhibits a typical thickener's behavior, with no noticeable variations of its thickening power regarding temperature. The VTF equation fits well the experimental data, with the exception of the PAMA-C above $60{ }^{\circ} \mathrm{C}$. The reduced slope observed above this temperature is linked to the swelling of polymers coils (10), which leads to an additional increase of the lubricant viscosity. The 
zoom between $60^{\circ} \mathrm{C}$ and $120^{\circ} \mathrm{C}$ in Fig. 1 (right) helps to clearly see this phenomenon. This latter is also slightly noticed for the PISH-S above $80^{\circ} \mathrm{C}$. The low shear viscosities at $40{ }^{\circ} \mathrm{C}$ and $100{ }^{\circ} \mathrm{C}$ are reported in Table 3 and allow a qualitative comparison of each additive.

Here, a rheological model can help to estimate the glass temperature transition $T_{g}(0)$ at atmospheric pressure. Typical viscosity for lubricants at the glass transition $\eta_{g}$ is assumed to be arbitrarily equal to $10^{12}$ Pa.s (21). The regression to VTF model was done on a smaller temperature range for the PAMA-C and PISH-S, below $80{ }^{\circ} \mathrm{C}$ for the first one and $100{ }^{\circ} \mathrm{C}$ for the second one, in order to improve the $T_{g}(0)$ determination without being impacted by the properties change at high temperature. No such consideration was done for other solutions. The values of $T_{g}(0)$ are detailed in Table 2. Direct measurements using differential scanning calorimetry (DSC) analyses were provided by TOTAL and are listed as well in Table 2. All $T_{g}(0)$ of polymer-thickened base oil solutions were close to the base oil value, regardless the determination process. This result was expected as all solutions were in diluted regimes and only a small amount of macromolecules was added (see concentrations in Table 1). The measured glass transition temperature of the studied products is close to the one deducted from the VTF model and validates thus the $T_{g}(0)$ calculations. Even though the rheological study at atmospheric pressure helped to distinguish a VII from a thickener, the investigation of the rheological behavior under severe conditions of pressure and shear stress is necessary to fully understand how the VIIs behave in the EHD contact.

\section{Pressure influence on viscosity index improvers' properties}

\section{Temperature and pressure dependence at low shear stress}

Series of tests were carried out on the high pressure viscometer at different temperatures and pressures to obtain the piezoviscous behavior of all solutions. Using directly the values of $T_{g}(0)$ previously determined, the data were fitted by a modified WLF-Yasutomi model (22) to get the variations of the low shear viscosity $\eta_{0}$ upon both temperature and pressure.

$$
\begin{aligned}
& \eta_{0}(T, p)=\eta_{g} \exp \left[\ln 10 \frac{-C_{1}\left(T-T_{g}(p)\right) F^{\prime}(p)}{C_{2}+\left(T-T_{g}(p)\right) F^{\prime}(p)}\right] \\
& T_{g}(p)=T_{g}(0)+A_{1} \ln \left(1+A_{2} p\right) \\
& F^{\prime}(p)=\left(1+b_{1} p\right)^{b_{2}}
\end{aligned}
$$


where $T_{g}(p)$ is the glass transition temperature at pressure $\mathrm{p}, F^{\prime}$ is the dimensionless relative thermal expansion of the free volume, $A_{1}, A_{2}, b_{1}, b_{2}, C_{1}, C_{2}$, are parameters determined by least mean square regressions of the experimental data.

The low shear viscosity $\eta_{0}$ is plotted against pressure for the base oil only, as all solutions exhibit a similar behavior where the viscosity highly increases with pressure, and is presented in semi-log scales in Fig. 2. Realistic initial conditions were used as initial parameters for the least mean square regressions of the experimental data ( $r m s$ deviation below 5\%). Details of parameter values and low shear viscosity at $40^{\circ} \mathrm{C}$ and $100^{\circ} \mathrm{C}$, at atmospheric pressure, can be found in Table 3 for the four different lubricants. The WLF-Yasutomi model Eq. [2] fits well the experimental data. The reciprocal asymptotic isoviscous pressure coefficient $\alpha^{*}$ is used to estimate the viscosity-pressure dependence at constant temperature. It is computed at $40{ }^{\circ} \mathrm{C}\left(\alpha_{40^{\circ} \mathrm{C}}{ }^{*}\right)$ and $100{ }^{\circ} \mathrm{C}\left(\alpha_{100^{\circ} \mathrm{C}}{ }^{*}\right)$ from the modified WLF-Yasutomi expression. This coefficient decreases with temperature for all products. However, it is interesting to notice that the PAMA-C coefficient variations differ from the other ones. Indeed, it is lower than the base oil coefficient whereas the PISH-S and OCP-LE coefficients are slightly bigger and get closer to the base oil coefficient at $100^{\circ} \mathrm{C}$. This different viscosity-pressure dependence is important to notice even though it is not fully understood yet.

\section{Thickening factor against pressure}

Temperature dependence of the low shear viscosity $\eta_{0}$ at atmospheric pressure enlightened the interesting VII effect of the PAMA-C additive. It is also possible to evaluate the VIIs efficiency by the viscosity index (VI), whose formula can be found below:

$$
V I=100 \frac{L-U}{L-H}
$$

where $U$ is the kinematic viscosity at $40^{\circ} \mathrm{C}$ of the oil whose VI is to be calculated, $L$ and $H$ are the kinematic viscosity at $40^{\circ} \mathrm{C}$ of reference oils, which have the same kinematic viscosity at $100^{\circ} \mathrm{C}$ as the oil under study, and have VIs respectively equal to 0 and 100.

Details of the viscosity index of each polymer-base oil solution can be found in Table 3 . These results confirm previous observations regarding the PAMA-C efficiency as this product presents the highest VI. However, it is not possible to know how the thickening power of the polymer varies with temperature. Indeed, the VI is a standard tool using reference oils, which are different from the base oil studied. This 
index is thus characteristic of the solution and it is difficult to highlight the contribution of the polymer itself. VII and thickeners cannot be then clearly distinguished by only looking at the viscosity index.

Even though information at ambient pressure is important, engine lubricants encounter severe conditions of pressure. It is then of primary interest to know how this latter affects the VII and thickener properties. The thickening factor $Q$ mentioned in the introduction (6) is another tool similar to the VI, to evaluate the variations of the polymer thickening power between $40{ }^{\circ} \mathrm{C}$ and $100{ }^{\circ} \mathrm{C}$ in the base oil. It is calculated at different pressures here:

$$
\begin{aligned}
& Q(p)=\frac{\eta_{s p}\left(\left[100^{\circ} \mathrm{C}\right], p\right)}{\left.\eta_{s p}\left(\left[40^{\circ} \mathrm{C}\right)\right], p\right)} \quad \text { where } \eta_{s p} \text { is the specific viscosity and is defined by } \\
& \eta_{s p}=\frac{\eta_{\text {solution }}-\eta_{\text {solvent }}}{\eta_{\text {solvent }}}
\end{aligned}
$$

When $Q=1$, the thickening power of the additive is the same regardless temperature (at the considered pressure). A thickening factor greater than 1 means that the thickening power is more important at high temperature, which is the desired effect of a VII. When $0<Q<1$, the thickening power is less significant at high temperature, which corresponds to the given definition of a thickener here. Fig. 3 shows the thickening factor plotted against pressure from atmospheric pressure up to $400 \mathrm{MPa}$, using the experimental data of the PAMA-C, PISH-S and OCP-LE base oil solutions obtained on the high pressure viscometer. Noticeably, the PAMA-C additive presents the highest thickening factor's values whatever the pressure. It is confirmed as the most efficient viscosity index improver studied here, whereas the OCP-LE is a thickener, as its thickening factor is below 1 all along the pressure range. The PISH-S is found to be a weak viscosity index improver, which validates previous comments (see Fig.1 and Table 3). The pressure doesn't impact the solutions properties, even though the PAMA-C thickening factor is enhanced at high pressures. This factor $Q$ is confirmed as a relevant indicator to quantify the VIIs actual performance.

\section{Impact of the molecular structure on the piezoviscous response}

As previously stated, the PAMA-C additive presented the most interesting properties (see Fig. 1 and Fig. 3 ). The hydrodynamic radius $R_{h}$ is a key parameter to explain how the structure of a polymer impacts its macroscopic properties. $R_{h}$ is defined as the radius of an equivalent hard sphere diffusing at the same rate as the molecule under observation. In reality, it represents the effective size of the polymer in its environment and varies with temperature, pressure and the nature of the solvent. Several methods exist 
to measure this parameter at atmospheric pressure, through direct measurements (for instance with dynamic light scattering) or by calculations using Flory equations or the Einstein's law. In their paper, Mary et al. applied the Einstein's law to high pressure (23):

$$
\begin{aligned}
& \eta_{\text {solution }}=\eta_{\text {solvent }}(1+2.5 \phi) \\
& \phi=\frac{c}{M} \frac{4}{3} \pi R_{h}^{3}
\end{aligned}
$$

where $\phi$ is the volume fraction, $c$ the concentration, $M$ the mass of one molecule $M=\frac{M_{w}}{N_{a}}$ where $N_{a}$ is the Avogadro number. Assumptions for the calculations are considering the polymer coils as rigid spheres and working with diluted solutions.

The hydrodynamic radius of the three polymers is calculated with high pressure viscometer measurements, using both the experimental data and the predicted viscosities from Eq. [2]. $R_{h}$ values are plotted as a function of temperature and pressure in Fig. 4. The uncertainty is equal to $10 \%$. For the sake of clarity, the error bars are plotted at atmospheric pressure only. The PAMA-C hydrodynamic radius increases by $79 \%$ from $25{ }^{\circ} \mathrm{C}$ to $120{ }^{\circ} \mathrm{C}$ at atmospheric pressure and by $115 \%$ at $200 \mathrm{MPa}$. The polymer coils obviously swell, confirming previous observation on the PAMA-C's efficiency (see Fig. 1). However, the swelling temperature cannot be precisely determined here and more experimental data between $40{ }^{\circ} \mathrm{C}$ and $100{ }^{\circ} \mathrm{C}$ are needed to be accurate. This phenomenon is also observed, but to a lesser extent for the PISH-S molecules: their radii increase by $20 \%$ between 25 and $120{ }^{\circ} \mathrm{C}$ at atmospheric pressure. In the case of the OCP-LE, $R_{h}$ decreases by $10 \%$ on the same temperature range.

The PAMA-C's peculiarity lies in its amphiphilic nature, having nonpolar carbonyls chains branched to a polar skeleton. Nonpolar solvent such as base oil dissolves easily nonpolar additives. The solubility is high, interactions between the solvent and the polymer segments are greater than intramolecular ones. The nonpolar additive can be then considered in "good solvent conditions", where the polymer coils are already fully swelled (24). On the contrary, if the solubility is low, the attraction between polymer segments themselves is preferred. The polymer is thus in "theta solvent" conditions, where the polymer chains tend to collapse and take a globule-like conformation. It is however accepted for the PAMA-C at low temperature and can be confirmed by hydrodynamic radius $R_{h}$ calculations using Flory equations in a theta solvent (see Table 4) at standard ambient temperature and pressure conditions.

Good solvent

$$
R_{h}=\frac{1}{1.56}\left(\frac{1}{\sqrt{6}} N^{0.6} l\right)
$$


Theta solvent

$$
R_{h}=\frac{1}{1.24}\left(\frac{1}{\sqrt{7}} N^{0.5} l\right)
$$

where $N$ is the number of monomers and $l$ the mean monomer size.

The deduced values of $R_{h}$ calculated from Eq. [6] are close to the calculated ones in theta solvent conditions. As the temperature increases, the solubility of the polymer in the base oil is improved. Thus, the Van der Waals interactions between the polymer coils and the solvent are enhanced. The polymer chains swell as the PAMA-C is in "good solvent" conditions at high temperature. The change of the solvent quality was confirmed by Covitch and Trickett using SANS (10), for the poly-alkyl methacrylate only. As regards the PISH-S and OCP-LE, the macromolecules are respectively in theta and good solvent conditions after looking at the calculated $R_{h}$ from Eq. [6]. These conditions of solvation were also observed in (10) for these kind of products.

When it comes to pressure, the hydrodynamic radius $R_{h}$ of the PAMA-C decreases by $20 \%$ at each temperature, whereas it remains steady for both PISH-S and OCP-LE (see Fig.4). This different pressure behavior could explain the particular reciprocal asymptotic isoviscous pressure coefficient of PAMA-C. The raise of pressure involves an increase of the density of the solvent, thus it is more difficult for the solute to be surrounded by solvent molecules, which explained the slight decrease for the PAMA-C. The molecular structure of polymers has then a strong effect on the solution properties upon temperature and pressure. However, these observations are made at low shear stress. The following section aims to understand how the rheological response is linked to the structure of polymer and how the VII efficiency is affected.

\section{SHEAR STRESS INFLUENCE ON VISCOSITY INDEX IMPROVER'S PROPERTIES}

The shear stress influence on the dynamic viscosity $\eta$ is quantified using the high pressure Couette rheometer. Many models exist to describe the dependence of viscosity upon shear rate or shear stress. Among them, the Carreau model (25) is widely used in EHL and has been improved many times over the years (26). The flow curves are here fitted by a Carreau-Yasuda model modified by Bair (27).

$$
\eta(T, p, \tau)=\eta_{2}(T, p)+\frac{\eta_{0}(T, p)-\eta_{2}(T, p)}{\left[1+\left(\frac{\tau}{G}\right)^{a}\right]^{\frac{\frac{1}{n}-1}{a}}}
$$


where $\tau(\mathrm{kPa})$ is the shear stress, $\eta_{2}$ (Pa.s) is the second Newtonian plateau viscosity, $G(\mathrm{kPa})$ is the apparent shear modulus, $a$ and $n$ are numerical parameters. The parameter $a$ determines the breadth of the transition from Newtonian to power-law regimes, whereas $n$ defines the slope.

The dynamic viscosity $\eta$ is then plotted against shear stress $\tau$ (see Fig. 5a); Fig. 5b); Fig 5c)). For the sake of clarity, only the results acquired at $30{ }^{\circ} \mathrm{C}$ for the polymers-thickened base oil solutions are shown in this paper. However, the general behavior is the same regardless the temperatures studied here (between $30{ }^{\circ} \mathrm{C}$ and $60{ }^{\circ} \mathrm{C}$ ). The effective shear modulus $G$ and the numerical parameters $a$ and $n$ determined by curve fitting, are detailed in Table 5. The viscosity (black line in Fig. 5) is here calculated from 0.01 to $1500 \mathrm{kPa}$ and using the modified Carreau-Yasuda expression. Different rheological responses were observed for each solution. Experimental results obtained on the high pressure viscometer (VHP) are also plotted as empty dots. Shear-thinning is here much more important for the PISH-S and the OCP-LE solutions (Fig. 5b) and Fig. 5c)) than for the PAMA-C one (Fig. 5a)). The viscosity decrease is about $50 \%$ for the PISH-S and $60 \%$ for the OCP-LE, whereas the viscosity of the PAMA-C only falls by $10 \%$.

\section{Relationship between the additive's structure and its rheology}

The different rheological behaviors are linked to the added polymer itself. However, the almost Newtonian behavior of PAMA-C was unexpected, especially as this solution has the highest polymer concentration and that the shear-thinning of lubricants comes from the addition of such product. Indeed, shear stress induces distortion and alignment of macromolecules resulting in a viscosity drop. Moreover, the amplitude of this behavior depends on the molecular weight of the polymer and its conformation (8, 9). In their paper, Mary et al. (23) distinguish conformation effect and size effect to explain the rheological responses of each polymer.

When it comes to molecules with comparable sizes, the conformation effect (the impact of their space disposal) can be investigated to explain the different rheology. Although they have very different molecular weights, PAMA-C and OCP-LE have comparable hydrodynamic radii at $25^{\circ} \mathrm{C}$ and $p_{a t m}$, and all along the experimental range investigated with the high pressure rheometer. However, the OCP-LE solution exhibits a strong shear-thinning whereas the PAMA-C solution is almost Newtonian. The OCPLE is a linear polymer, whereas the PAMA-C is a comb one. This latter presents intramolecular interactions, resulting in a much less flexible macromolecule compared to the OCP-LE. PAMA-C molecules are thus less shear-sensitive, explaining their almost Newtonian behavior. 
On the other hand both PAMA-C and PISH-S additives have conformation implying strong steric hindrance. However, the PISH-S solution presents an important shear-thinning. The PISH-S hydrodynamic radius is larger than the PAMA-C at each temperature (see Table 4). According to (9), shearing implies a drop of viscosity on larger molecules for comparable intramolecular bonds, which explains then these different behaviors.

\section{Limits of the Carreau-Yasuda model}

Using high pressure rheological measurements, thickeners and viscosity index improvers could then be distinguished. The PAMA-C was highlighted as the most interesting VII, its properties enhanced by the swelling of its polymer coils above $80^{\circ} \mathrm{C}$. This behavior is difficult to model using the Carreau-Yasuda relationship (Eq. [7]). Indeed this model requires many parameters to be determined and relies on rather strong assumptions. In particular, the shear modulus $G$ and the ratio between the first Newtonian plateau (i.e. the low shear stress viscosity) $\eta_{0}$ and the second Newtonian plateau $\eta_{2}$ are both kept constant at any temperature and pressure. As mentioned previously, the PAMA-C molecules are fully swelled at high temperature. They would be then more shear-sensitive and a shear-thinning response may be expected. The ratio between the two plateaus may be then affected by the temperature in case of polymers showing effective VII's properties. As these VIIs are more and more used in lubricants formulation, the current rheological models might be inaccurate and lead to unsatisfactory film thickness predictions. An alternative approach should then be considered.

\section{ZHANG MODEL FOR LOW VISCOELASTIC SOLUTIONS}

Typical models used in elastohydrodynamic lubrication - as the Carreau-Yasuda one - are based on molecular theory for the real viscosity. In a recent paper (28), Zhang took a different approach and proposed to describe the shear rate influence on viscosity based on a Maxwell viscoelastic-like response. This latter is a mechanical model in which a purely viscous damper and a purely elastic spring are connected in series and gives an equivalent viscosity. All existing models consider that only the real viscosity $\eta_{\text {real }}$ - which corresponds to viscosity of the first Newtonian plateau $\eta_{0^{-}}$changes under the shear stress influence without taking into account that the elastic modulus - thus the elastic shear modulus $G$ - may also change.Zhang (28) explained that the measured viscosity $\eta$ deviates from the prediction of the linear Maxwell model because of the elasticity variations and not the variations of viscosity. This results in a linear dependence of the elastic modulus on the shear strain rate, pressure and temperature. The resulting expression is adapted to $T, p$ and $\dot{\gamma}$ shown below: 


$$
\begin{aligned}
& \eta(T, p, \dot{\gamma})=\frac{\eta_{0}(T, p)}{1+\left[\frac{\eta_{0}(T, p) \dot{\gamma}}{2 . G(T, p, \dot{\gamma})}\right]^{2}} \\
& G(T, p, \dot{\gamma})=G_{0}+\beta \eta_{0}(T, p) \dot{\gamma}
\end{aligned}
$$

where $\dot{\gamma}\left(\mathrm{s}^{-1}\right)$ corresponds to the shear strain rate, $G(\mathrm{kPa})$ is the equivalent shear modulus, $G_{0}(T, p)(\mathrm{kPa})$ is the equivalent shear modulus at low shear strain rate, $\beta$ is a dimensionless constant.

Taking into account the variations of the shear modulus is an interesting approach because it induces, without introducing any supplementary parameter, a second Newtonian plateau $\eta_{2}$ which is known to be hard to fully characterize in experiments. The linear dependence was chosen here because it is the first order of approximation, widely used in both science and engineering. The Zhang model only applies though to solutions which show a strong elastic deformation, meaning with an important shear-thinning response. The maximum elastic deformation is dependent only on the parameter $\beta$. According to the author, this latter should be less than 0.5 to model a significant viscoelastic behavior. This implies that the measured viscosity $\eta$ should at least be reduced by half, which is not viscoelastic the case for most of the solutions studied. The extension to low-solutions such as the simplified lubricants studied here needs to be verified beforehand. Hence, all values resulting from Zhang model are compared to CarreauYasuda predictions in order to validate the application to less viscoelastic products. The experimental data and calculated viscosities from Zhang model are shown in Fig. 6a), Fig. 6b) and Fig. 6c) for all polymer-thickened base oil solutions. The dynamic viscosity $\eta$ is here plotted against shear strain rate $\dot{\gamma}$ at $30{ }^{\circ} \mathrm{C}$ and from $200 \mathrm{MPa}$ to $400 \mathrm{MPa}$. The viscosity is then calculated from 10 to $1 \times 10^{6} \mathrm{~s}^{-1}$. The initial shear modulus $G_{0}$ and the dimensionless parameter $\beta$ determined by curve fitting to the Zhang model are shown in Table 5. Even though the solutions studied don't present a strong viscoelastic behavior, the results obtained are satisfying and in accordance with previous conclusions.

In this paragraph the viscosities modeled with Zhang's expression are compared to the Carreau-Yasuda predictions in order to validate their application to low-viscoelastic solutions. To easily compare both models, the master curves are presented for the PAMA-C, PISH-S and OCP-LE solutions (Fig. 7, Fig. 8, Fig. 9), where all the viscosities at each condition of temperature and pressure overlap on a single curve. The advantages of the Zhang model and limits of the modeling are illustrated by these graphs.

As can be seen in Fig. 7, the calculated viscosity using the Zhang model is in good agreement with the Carreau-Yasuda prediction and results are promising for VII application. The regression made with Zhang equation gave results as good as those obtained using the Carreau-Yasuda one (see rms Table 5). However, no assumption was made on the second Newtonian plateau $\eta_{2}$, which is an advantage. The 
Zhang viscosities are in a better accordance with the high pressure viscometer results $\eta_{0, \exp }$ (at low shear stress), as can be seen from Table 5. The shear modulus prediction for the PAMA-C solution is quite different: this result was expected as the Zhang model is here applied to an almost Newtonian solution. It is not possible to say which $G$ prediction is the most accurate, as the shear modulus can't be determined experimentally yet for the solutions studied. However, $G_{0}$ calculated using Zhang expression is physically more coherent. Indeed, its variations upon temperature, pressure and shear rate are taken into account. Moreover, the higher the shear modulus, the less shear-sensitive the macromolecule. The PAMA-C $G_{0}$ is the highest, as this solution doesn't shear-thin (see Table 5) and its macromolecules are not flexible. The Zhang model needs then less parameters, whose physical significations are better defined. As the limits of the Carreau-Yasuda model mainly concerned actual VII (such as the PAMAC), these results are really promising for similar applications.

Regarding the PISH-S and OCP-LE results (see Fig. 8 and Fig.9), the experimental data fit well with both expressions though extrapolated viscosities are slightly different. The results show indeed different behaviors according to the chosen model for the OCP-LE solution. A second Newtonian plateau is always present when fitting data with Zhang equation due to its construction whereas an important shearthinning response is observed with Carreau-Yasuda predictions. This discrepancy highlights the limit of this particular modeling due to experimental limitations. In most cases, a second Newtonian plateau or its onset should be expected at high shear stress, but it can't be reached experimentally yet. It is thus not possible to say if the viscosity drop is over or underestimated here.

\section{SUMMARY}

Experiments carried out on high pressure devices provided the rheological response of polymer-base oil mixtures over a large range of temperature, pressure and shear stress.

The dependence of viscosity on temperature and pressure was fully and accurately characterized for all solutions. True viscosity index modifiers can be distinguished from thickeners in realistic engine conditions by considering the thickening factor Q (rather than the VI) and the variations of their $R_{h}$. The calculation of the latter was performed thanks to the Einstein's law applied to high pressure conditions. The PAMA-C additive was found to be the more efficient viscosity index improver studied here. A noticeable increase of its hydrodynamic radius was observed, confirming the polymer coil expansion with temperature and explaining the variations of its thickening factor. Its particular viscosity-pressure dependence was also highlighted and linked to its piezoviscous coefficient. 
Compared with the PAMA-C, the PISH-S solution exhibited a rather limited VII effect, according its lower factor $Q$ and the evolution of its hydrodynamic radius. In this work, the OCP-LE was considered as a thickener.

The shear stress dependence of viscosity was then studied experimentally and predicted by different rheological models. All the models displayed strong agreement with the experimental data. Different rheological responses were highlighted regarding each considered additive. An unexpected Newtonian behavior was noticed for the PAMA-C solution, within the range investigated in this paper. The effect of shear stress on hydrodynamic radius was then investigated, considering both effect of the macromolecules' sizes and conformations. At comparable sizes, conformation effects are predominant. A linear molecule - like the OCP-LE - is more shear-sensitive than a comb polymer - like the PAMA-C - because of its strong steric hindrance. At low temperature, both PISH-S and PAMA-C polymers are difficult to stretch because of their conformation. However, PISH-S's molecules are bigger than PAMAs' ones, are then more stretchable and affected by shear stress.

Nonetheless, PAMA-C's macromolecules swelled at high temperature. Once fully swelled, the PAMA$\mathrm{C}$ molecules should be more shear-sensitive and a shear-thinning response may be expected. The ratio between the two Newtonian plateaus, kept constant in the Carreau-Yasuda model, may be affected by the temperature and pressure. The Zhang model was then applied to solutions with low viscoelastic behaviors. Results were promising and applying this model to VIIs could be a good alternative to the Carreau-Yasuda one.

\section{ACKNOWLEDGMENT}

The authors would like to thank TOTAL for its financial and technical support. We are also grateful to

Prof. C. Chassenieux, Prof. J.-F. Tassin and J. Dorenge from the IMMM laboratory (Université du Maine, Le Mans, France) for providing the SEC results. 


\section{REFERENCES}

(1) Holmberg, K., Andersson, P., Erdemir, A. (2012). Global energy consumption due to friction in passenger cars. Tribology International, 47, pp. 221-234.

(2) Bhattacharya, P., Ramasamy, U. S., Krueger, S., Robinson, J. W., Tarasevich, B. J., Martini, A., Cosimbescu, L. (2016). Trends in Thermo-Responsive Behavior of Lipophilic Polymers. Industrial \& Engineering Chemistry Research, 55(51), pp. 12983-12990

(3) Ghosh, P., Das, M. (2014). Study of the influence of some polymeric additives as viscosity index improvers and pour point depressants-synthesis and characterization. Journal of Petroleum Science and Engineering, 119, pp. 79-84.

(4) Canter, N. (2011). Viscosity index improvers. Tribology \& Lubrication Technology, 67(9), pp. 10.

(5) ASTM D2270 -Calculating viscosity index from kinematic viscosity at 40 and $100^{\circ} \mathrm{C}$.

(6) Singh, H., Gulati, I. B. (1987). Influence of base oil refining on the performance of viscosity index improvers. Wear, 118(1), pp. 33-56.

(7) Fox, M. F. (2010). Chemistry and technology of lubricants (Vol. 107115). R. M. Mortier, S. T. Orszulik (Eds.). London: Springer, ISBN 97814020 8662-5

(8) Barnes, H. A., Hutton, J. F., Walters, K. (1989). An introduction to rheology (Vol. 3). Elsevier, ISBN 0444874690

(9) Schulz, D. N., Glass, J. E. (1991). Polymers as rheology modifiers. American Chemical Society, ACS Symp. Ser., ISBN 0841220093

(10) Covitch, M. J., Trickett, K. J. (2015). How polymers behave as viscosity index improvers in lubricating oils. Advances in Chemical Engineering and Science, 5(02), pp. 134.

(11) Bair, S. S. (2007). High pressure rheology for quantitative elastohydrodynamics (Vol. 54). Elsevier, ISBN 9780444522436

(12) Habchi, W., Vergne, P., Bair, S., Andersson, O., Eyheramendy, D., Morales-Espejel, G.E. (2010). Influence of Pressure and Temperature Dependence of Thermal Properties of a Lubricant on the behaviour of Circular TEHD Contacts. Tribology International, 43(10), pp. 1842-1850.

(13) Doki-Thonon, T., Fillot, N., Morales Espejel, G.E., Querry, M., Philippon, D., Devaux, N., Vergne,P. (2013). A dual experimental / numerical approach for film thickness analysis in TEHL spinning skewing circular contacts. Tribology Letters, 50(1), pp. 115-126.

(14) Krupka, I., Kumar, P., Bair, S., Khonsari, M. M., Hartl, M. (2010). The effect of load (pressure) for quantitative EHL film thickness. Tribology Letters, 37(3), pp. 613-622. 
(15) ASTM D4741 - Measuring viscosity at high temperature and high shear rate by tapered-plug viscometer

(16) Bair, S., Winer, W. O. (1980). Some observations on the relationship between lubricant mechanical and dielectric transitions under pressure. Journal of Lubrication Technology, 102(2), pp. 229-234.

(17) Bair, S., Winer, W. O. (1993). A new high-pressure, high-shear stress viscometer and results for lubricants. Tribology Transactions, 36(4), pp. 721-725.

(18) Andrade, E. D. C. (1930). The Viscosity of Liquids. Nature, 125, pp. 309-310.

(19) Eyring, H. (1936). Viscosity, plasticity, and diffusion as examples of absolute reaction rates. The Journal of Chemical Physics, 4(4), pp. 283-291.

(20) Angell, C. A. (1995). Formation of glasses from liquids and biopolymers. Science, 267(5206), pp. 1924.

(21) Barlow, A. J., Erginsav, A., Lamb, J. (1969, April). Viscoelastic relaxation in liquid mixtures. In Proceedings of the Royal Society of London A: Mathematical, Physical and Engineering Sciences, 309 (1499), pp. 473-496

(22) Bair, S., Mary, C., Bouscharain, N., Vergne, P. (2013). An improved Yasutomi correlation for viscosity at high pressure. Proceedings of the Institution of Mechanical Engineers, Part J: Journal of Engineering Tribology, 227(9), pp. 1056-1060

(23) Mary, C., Philippon, D., Lafarge, L., Laurent, D., Rondelez, F., Bair, S., Vergne, P. (2013). New insight into the relationship between molecular effects and the rheological behavior of polymer-thickened lubricants under high pressure. Tribology Letters, 52(3), pp. 357-369.

(24) Flory, P. J. (1953). Principles of polymer chemistry. Cornell University Press, ISBN 0801401348

(25) Carreau, P. J. (1972). Rheological equations from molecular network theories. Transactions of The Society of Rheology (1957-1977), 16(1), pp. 99-127.

(26) Tanner, R.I., (2000) Engineering Rheology, second edition, Oxford Univ. Press, Oxford, ISBN 019 8564732

(27) Bair, S. (2004). A rough shear-thinning correction for EHD film thickness. Tribology Transactions, 47(3), pp. 361-365.

(28) Zhang, B. (2016). A Novel Model of the Equivalent Viscosity of Simple Viscoelastic Fluids. Journal of Tribology, 138(2), 021802. 


\section{NOMENCLATURE}

$a, n$

$A_{1}, A_{2}, b_{1}, b_{2}, C_{1}, C_{2}$,

$C$

$D_{F}$

$F^{\prime}$

G

$G_{0}$

$l$

M

$M_{w}$

$N$

$N_{a}$

$p$

$Q$

$R_{h}$

$T$

$T_{\infty}$

$T_{g}(0)$

$T_{g}(p)$

$\alpha^{*}$

$\alpha_{40^{\circ} \mathrm{C}^{*}}$

$\alpha_{100^{\circ} \mathrm{C}}{ }^{*}$

$\beta$

$\dot{\gamma}$

$\eta$

$\eta_{0}$

$\eta_{0, \exp }$

$\eta_{2}$

$\eta_{\infty}$

$\eta_{g}$

$\eta_{\text {real }}$

$\eta_{\text {solution }}$

$\eta_{\text {solvent }}$

$\eta_{s p}$

$\tau$

$\phi$
Carreau-Yasuda parameters

WLF parameters

Concentration in weight of active material $\left(\mathrm{g}^{\mathrm{c}} \mathrm{cm}^{-3}\right)$

Fragility parameter

Dimensionless relative thermal expansion of the free volume

Shear modulus $(\mathrm{kPa})$

Equivalent shear modulus at low shear rate $(\mathrm{kPa})$

Mean monomer size $(\mathrm{cm})$

Mass of one molecule (g)

Weight average molecular mass $\left(\mathrm{g} \cdot \mathrm{mol}^{-1}\right)$

Number of monomers

Avogadro number $\left(\mathrm{mol}^{-1}\right)$

Pressure (MPa)

Thickness factor

Hydrodynamic radius (nm)

Temperature $\left({ }^{\circ} \mathrm{C}\right)$

Vogel temperature $\left({ }^{\circ} \mathrm{C}\right)$

Glass transition temperature at atmospheric pressure $\left({ }^{\circ} \mathrm{C}\right)$

Glass transition temperature at pressure $\mathrm{p}\left({ }^{\circ} \mathrm{C}\right)$

Reciprocal asymptotic isoviscous pressure coefficient $\left(\mathrm{GPa}^{-1}\right)$

Piezoviscous coefficient at $40{ }^{\circ} \mathrm{C}\left(\mathrm{GPa}^{-1}\right)$

Piezoviscous coefficient at $100{ }^{\circ} \mathrm{C}\left(\mathrm{GPa}^{-1}\right)$

Zhang model dimensionless constant

Shear strain rate $\left(\mathrm{s}^{-1}\right)$

Dynamic viscosity (Pa.s)

Low shear viscosity (Pa.s)

High pressure viscometer experimental viscosity (Pa.s)

Viscosity on the second Newtonian plateau (Pa.s)

Viscosity extrapolated to infinite temperature (Pa.s)

Viscosity at the glass transition (Pa.s)

Real viscosity in Maxwell model (Pa.s)

Viscosity of the polymer-thickened base oil solution (Pa.s)

Viscosity of the base oil (Pa.s)

Specific viscosity

Shear stress $(\mathrm{kPa})$

Volume fraction 

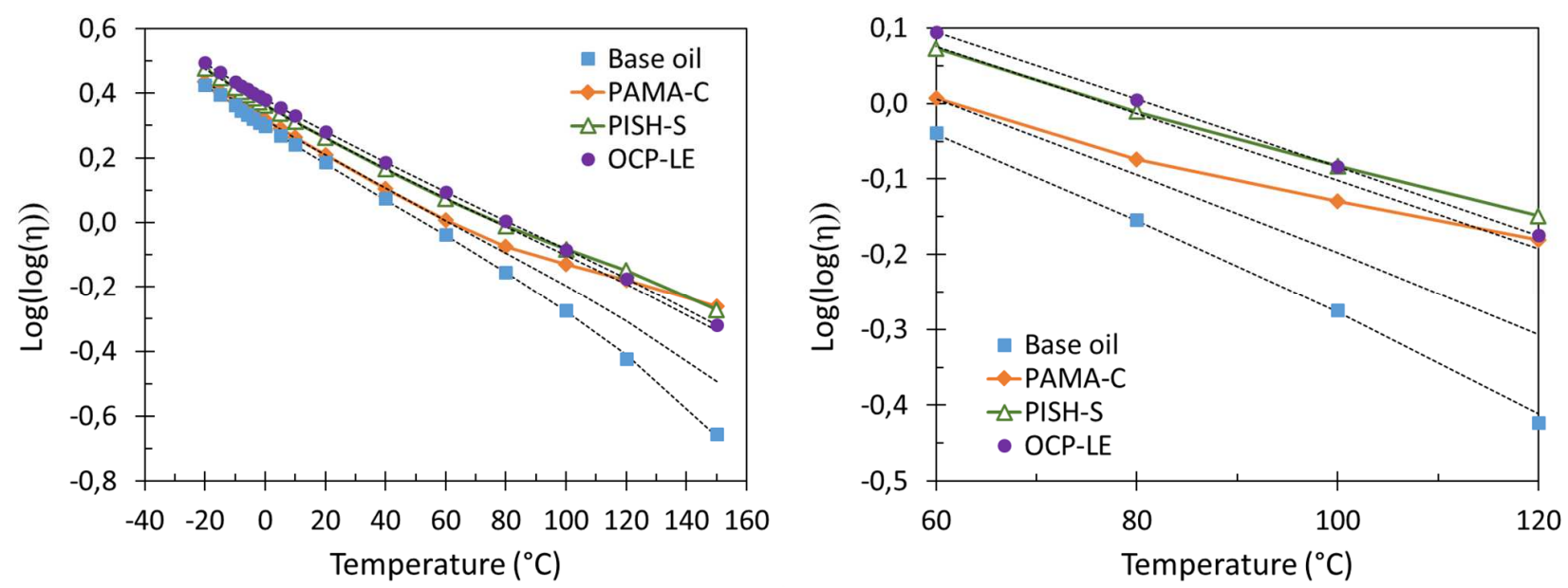

Fig. 1: Low shear viscosity, expressed as $\log \left(\log \left(\eta_{0}\right)\right)$, of the base oil, PAMA-C, PISH-S and OCP-LE solutions versus temperature and fitted by the VTF expression plotted as the dotted black line - between $-40^{\circ} \mathrm{C}$ and $160^{\circ} \mathrm{C}$ (left) and zoomed between 60 and $120^{\circ} \mathrm{C}$ (right) to highlight the slop discrepancy of PAMA-C and PISH-S.

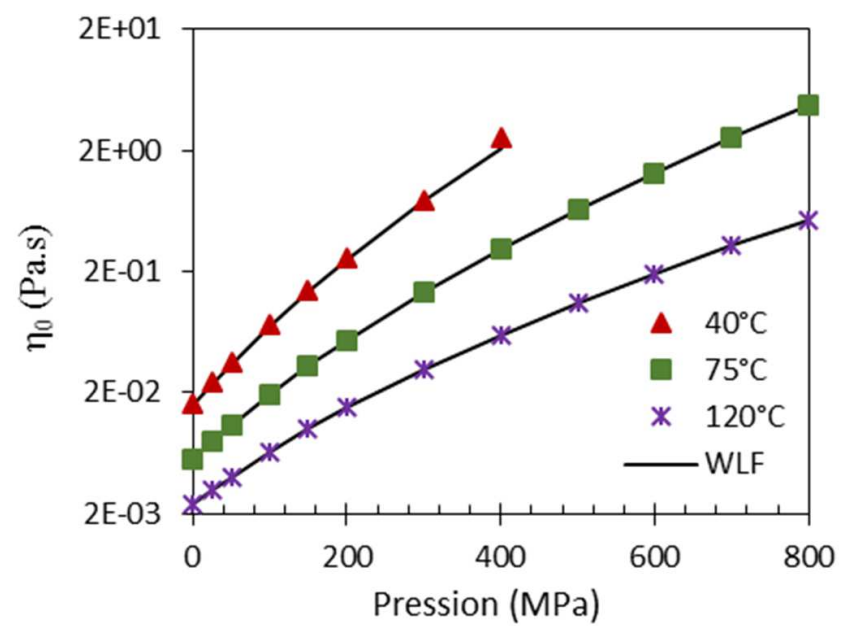

Fig. 2: Viscosity-pressure dependence of the base oil (experimental data as colored dots) at different temperatures and regressed to the modified WLF-Yasutomi expression (black line) 


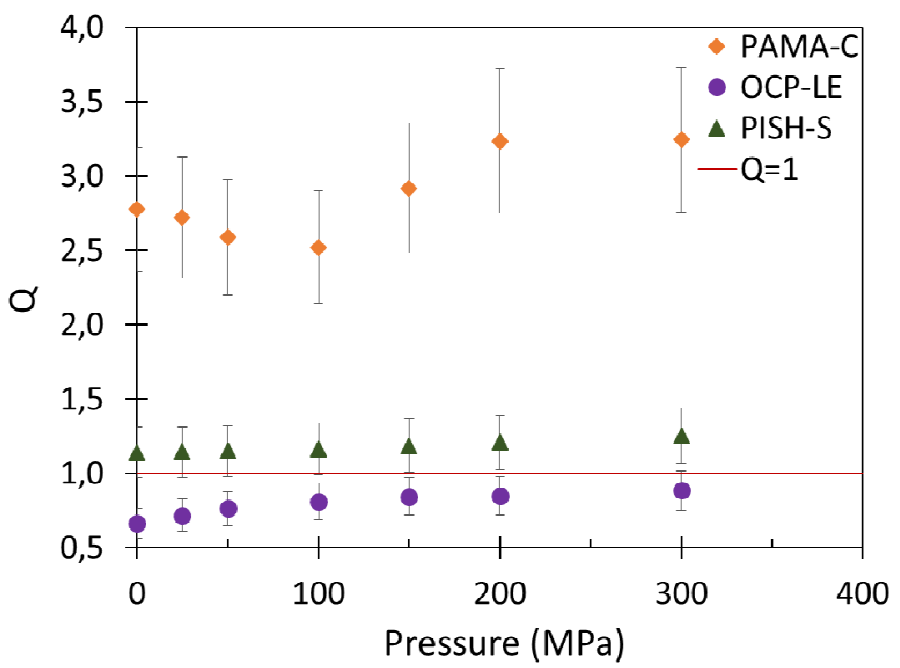

Fig. 3: Thickening factor $Q$ versus pressure for PAMA-C, PISH-S and OCP-LE 

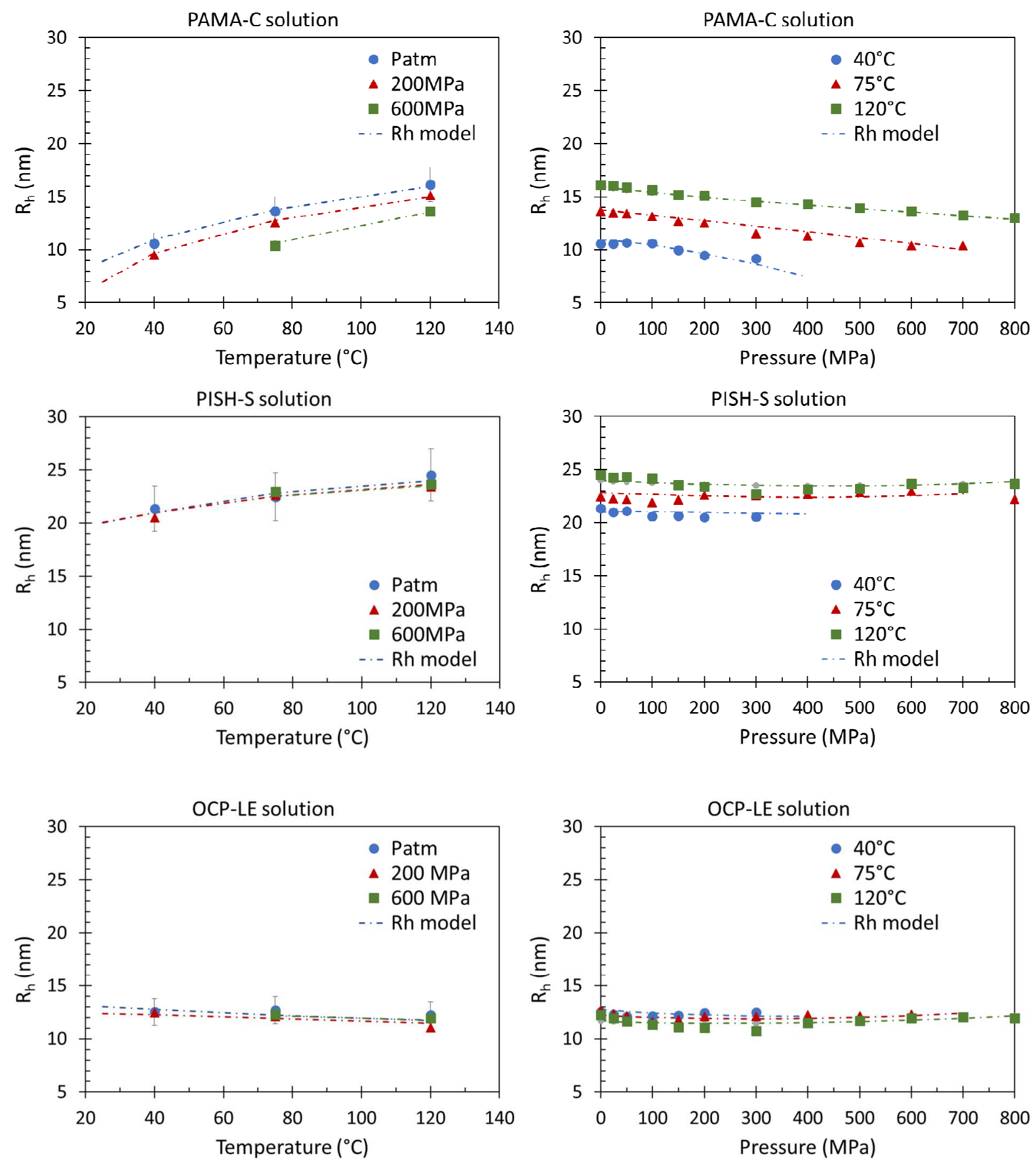

Fig. 4: Hydrodynamic radius variations versus temperature (left) and pressure (right) for all polymerbase oil solutions $-R_{h}$ determined from experimental values plotted as color dots and calculated with Eq [2] as dotted lines. 

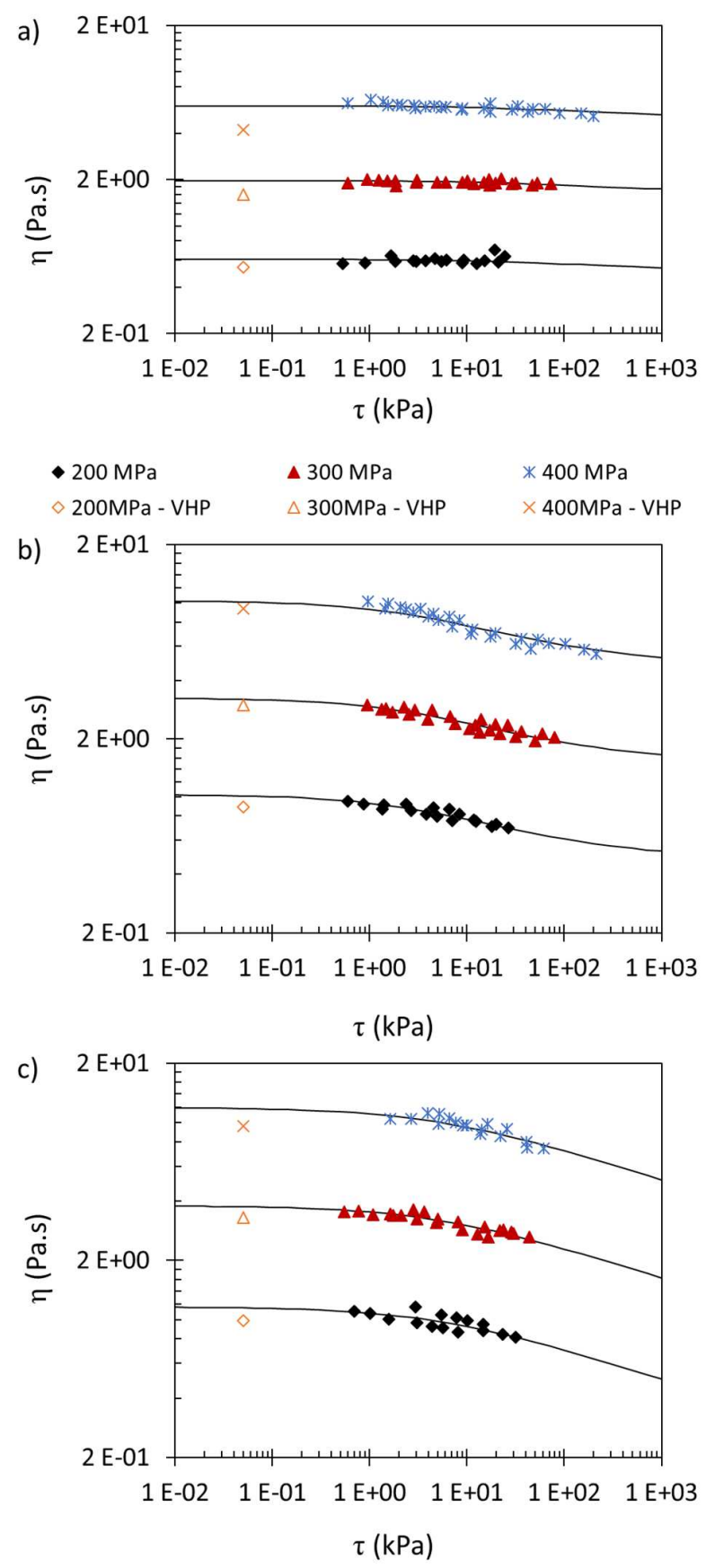

Fig. 5: Viscosity of the polymer-base oil solution versus shear stress at $30{ }^{\circ} \mathrm{C}$ and different pressures, and fitted to the modified Carreau-Yasuda expression (black line) - the a), b), c) figures correspond respectively to the PAMA-C, PISH-S and OCP-LE solutions 


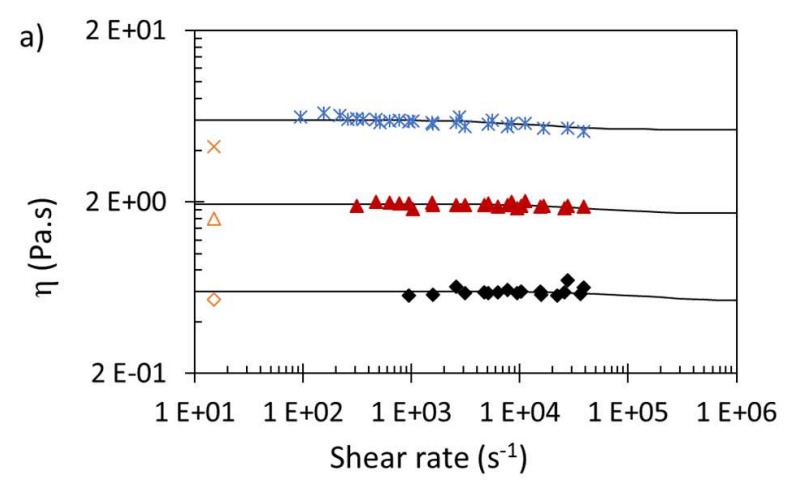

$\begin{array}{lll}-200 \mathrm{MPa}-\text { Low } & \triangle 300 \mathrm{MPa}-\text { Low } & * 400 \mathrm{MPa}-\text { Low } \\ \diamond 200 \mathrm{MPa}-\mathrm{VHP} & \triangle 300 \mathrm{MPa}-\mathrm{VHP} & \times 400 \mathrm{MPa}-\mathrm{VHP}\end{array}$
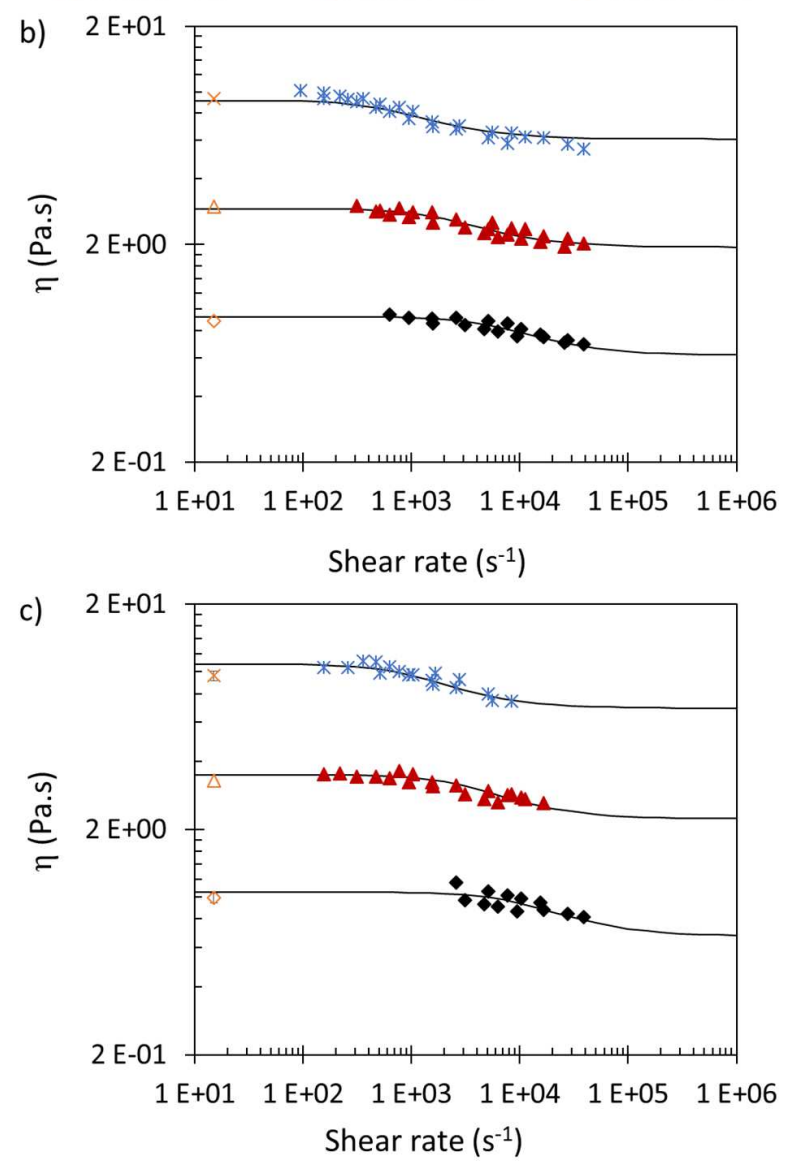

Fig. 6: Viscosity of the polymer-base oil solution versus shear stress at $30{ }^{\circ} \mathrm{C}$ and different pressures, and fitted to the Zhang equation (black line) - the a), b), c) figures correspond respectively to the PAMAC, PISH-S and OCP-LE solutions 


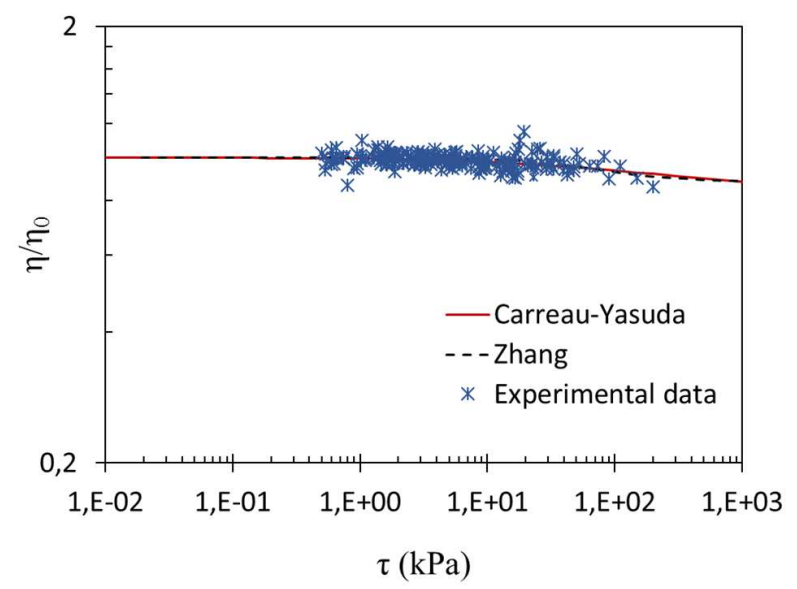

Fig. 7: Comparison of Carreau-Yasuda predictions and Zhang results for the PAMA-C solution by looking at the master curve and experimental results obtained at different temperatures and pressures

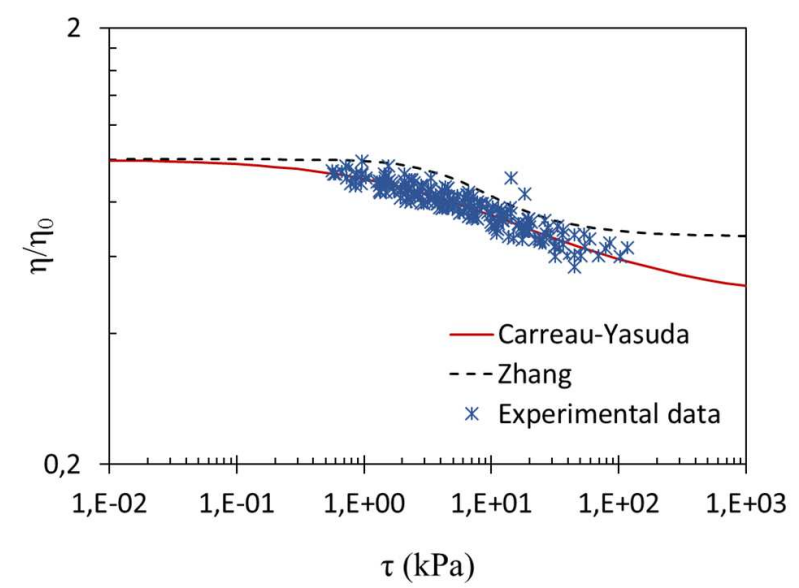

Fig. 8: Comparison of Carreau-Yasuda predictions and Zhang results for the PISH-S solution by looking at the master curve and experimental results obtained at different temperatures and pressures

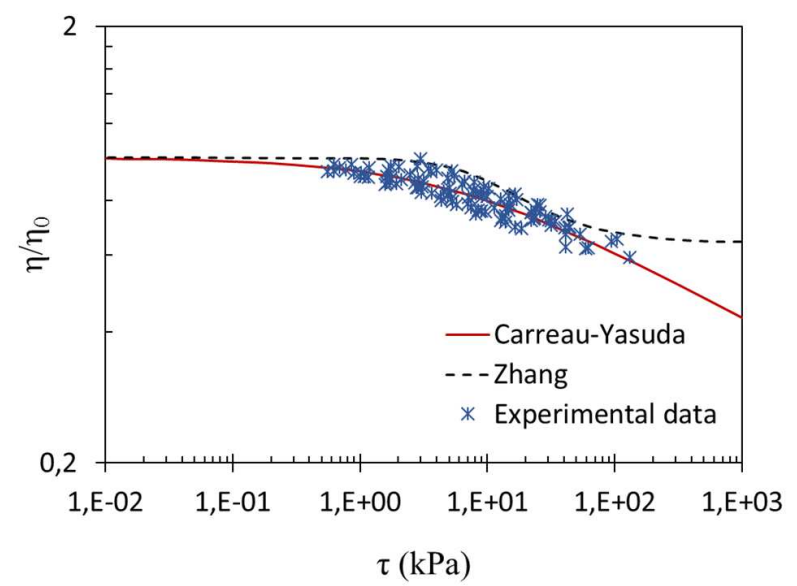

Fig. 9: Comparison of Carreau-Yasuda predictions and Zhang results for the OCP-LE solution by looking at the master curve and experimental results obtained at different temperatures and pressures 
TABLES

\begin{tabular}{|c|c|c|c|}
\hline Polymer & PAMA & PISH & $\mathrm{OCP}$ \\
\hline Chemical formula & $\left.\begin{array}{r}{\left[\mathrm{CH}_{2}-\mathrm{C}\right.} \\
\mathrm{C} \\
\mathrm{C} \\
1 \\
\mathrm{O}\end{array}\right]=0$ & & \\
\hline Name & PAMA - C & PISH-S & OCP-LE \\
\hline Molecular structure & ${ }^{\text {Comb }}$ & Star & Linear \\
\hline$M_{w}(\mathrm{~g} / \mathrm{mol})$ & 678000 & 689600 & 87300 \\
\hline PDI & 3.16 & 1.10 & 1.60 \\
\hline $\begin{array}{l}\text { Concentration of } \\
\text { active ingredient } \\
\left(\%_{w / w}\right)\end{array}$ & 3.36 & 1.18 & 1.28 \\
\hline
\end{tabular}

Table 1: Main characteristics of the polymers studied

\begin{tabular}{|l|l|l|l|l|}
\hline$T_{g}(0)\left({ }^{\circ} \mathrm{C}\right)$ & Base oil & PAMA-C & PISH-S & OCP-LE \\
\hline by VTF & -90.5 & -92.4 & -91.9 & -92.7 \\
\hline by DSC & -94.8 & -95.5 & -94.4 & -94.6 \\
\hline
\end{tabular}

Table 2: Extrapolated (from VTF model) and measured by DSC glass transition temperatures at atmospheric pressure 


\begin{tabular}{|c|c|c|c|c|}
\hline Material & Base oil & $\begin{array}{l}\text { Base oil + PAMA- } \\
\text { C }\end{array}$ & Base oil + PISH-S & $\begin{array}{l}\text { Base oil + OCP- } \\
\text { LE }\end{array}$ \\
\hline$A_{1}\left({ }^{\circ} \mathrm{C}\right)$ & 61.09 & 55.46 & 106.67 & 161.31 \\
\hline$A_{2}\left(\mathrm{GPa}^{-1}\right)$ & 0.978 & 0.866 & 0.498 & 0.320 \\
\hline$b_{1}\left(\mathrm{GPa}^{-1}\right)$ & 6.49 & 7.72 & 7.34 & 6.15 \\
\hline$b_{2}$ & -0.499 & -0.550 & -0.515 & -0.487 \\
\hline$C_{1}$ & 16.18 & 15.54 & 15.72 & 16.11 \\
\hline$C_{2}\left({ }^{\circ} \mathrm{C}\right)$ & 22.57 & 17.99 & 21.04 & 26.02 \\
\hline$\eta_{g}(\mathrm{~Pa} . \mathrm{s})$ & $10^{12}$ & $10^{12}$ & $10^{12}$ & $10^{12}$ \\
\hline$T_{g}(0)\left({ }^{\circ} \mathrm{C}\right)$ & -90.5 & -92.4 & -91.9 & -92.7 \\
\hline $\operatorname{rms}(\%)$ & 3.31 & 4.19 & 3.82 & 2.83 \\
\hline$\alpha_{40^{\circ} C^{*}}\left(\mathrm{GPa}^{-1}\right)$ & 14.7 & 14.4 & 15.1 & 14.7 \\
\hline$\alpha_{100^{\circ} \mathrm{C}}{ }^{*}\left(\mathrm{GPa}^{-1}\right)$ & 10.1 & 9.9 & 10.4 & 10.3 \\
\hline $\begin{array}{l}\eta_{0}\left(40^{\circ} \mathrm{C}, \text { Patm }\right) \\
\text { (mPa.s) }\end{array}$ & 16.36 & 21.28 & 30.33 & 34.87 \\
\hline $\begin{array}{l}\eta_{0}\left(100^{\circ} \mathrm{C}, \mathrm{Patm}\right) \\
\text { (mPa.s) }\end{array}$ & 3.33 & 6.11 & 6.88 & 6.42 \\
\hline VI & 148 & 306 & 235 & 187 \\
\hline
\end{tabular}

Table 3: Parameters of the modified WLF-Yasutomi model (21), reciprocal asymptotic isoviscous pressure coefficients computed from Eq. [2] and VI of all solutions.

\begin{tabular}{|l|l|l|l|l|}
\hline Rh $(\mathrm{nm})$ & PAMA-C & PISH-S & OCP-LE \\
\hline $\begin{array}{l}\text { Einstein's law } \\
\left(25-120^{\circ} \mathrm{C} ; \text { Patm }\right)\end{array}$ & $8.9-16.0$ & $20.1-24.0$ & $13.0-11.5$ \\
\hline $\begin{array}{l}\text { Flory equations } \\
\left(25^{\circ} \mathrm{C} ; \text { Patm }\right)\end{array}$ & Good solvent & 23.0 & 63.3 & 8.8 \\
\cline { 2 - 5 } & Theta solvent & 11.8 & 23.3 & 4.7 \\
\hline
\end{tabular}

Table 4: Hydrodynamic radius at ambient pressure derived from Einstein and Flory's equations. For Flory predictions, the solvent quality which does not correspond to the experiments are reported in greyed cells.

\begin{tabular}{|l|l|l|l|l|}
\hline \multicolumn{2}{|c|}{ Material } & PAMA-C solution & PISH-S solution & OCP-LE solution \\
\hline \multirow{5}{*}{ Carreau-Yasuda } & $G(\mathrm{kPa})$ & 8.27 & 4.06 & 4.99 \\
\cline { 2 - 5 } & $a$ & 0.988 & 0.687 & 0.654 \\
\cline { 2 - 5 } & $n$ & 0.975 & 0.705 & 0.864 \\
\cline { 2 - 5 } & rms $(\%)$ & 3.7 & 4.6 & 5.0 \\
\cline { 2 - 5 } & Gap to $\eta_{0, \exp }(\%)$ & $10-26$ & $12-20$ & $19-25$ \\
\hline \multirow{3}{*}{ Zhang } & $G_{0}(\mathrm{kPa})$ & 50.17 & 4.56 & 8.35 \\
\cline { 2 - 5 } & $\beta$ & 1.33 & 0.71 & 0.63 \\
\cline { 2 - 5 } & rms $(\%)$ & 3.6 & 3.5 & 5.1 \\
\cline { 2 - 5 } & Gap to $\eta_{0, \exp }(\%)$ & $10-24$ & $3-9$ & $5-16$ \\
\hline
\end{tabular}

Table 5: Parameters of the Carreau-Yasuda and Zhang models 